\title{
Cyclic Force Applied to FAs Induces Actin Recruitment Depending on the Dynamic Loading Pattern
}

\author{
Y. Ueki ${ }^{1,2}$, N. Sakamoto ${ }^{1}$ and M. Sato*,1,3 \\ ${ }^{I}$ Graduate School of Engineering, Tohoku University; ${ }^{2}$ Japan Society for the Promotion of Science, ${ }^{3}$ Graduate School of \\ Biomedical Engineering, Tohoku University, Japan
}

\begin{abstract}
Mechanical forces acting on focal adhesions (FAs) are believed to be an important determinant for cytoskeletal reorganization. However, the effect of the temporal pattern of forces on cellular responses has not been elucidated. In the present study, we examined the responses of FAs to locally-applied cyclic forces. Magnetic micro beads coated with fibronectin were attached to the apical surface of endothelial cells and continuous or cyclic forces at frequencies of 0.1-10 $\mathrm{Hz}$ with duty cycles of $0-100 \%$ were applied to the beads using a newly developed electromagnetic tweezer. A significant increase in actin recruitment around the beads was observed when cyclic forces at $1-2 \mathrm{~Hz}$ and $25-50 \%$ duty cycles were applied. This tendency disappeared upon modification of myosin activity. These results indicate that the sensitivity to temporal patterns of forces is detemined by the viscoelastic properes of FAs and depends on myosin activity.
\end{abstract}

Keywords: Focal Adhesion, mechanotransduction, endothelial cell, electromagnetic tweezwer.

\section{INTRODUCTION}

Vascular cells, such as endothelial cells (ECs) and smooth muscle cells (SMCs), are exposed to cyclic stretch (CS) due to pulsatile blood flow. Previous in vitro studies reported that this mechanical stimulus induces the cells to reorient and become almost perpendicular to the direction of the stretch associated with the development and alignment of actin stress fibers [1-3]. Such remodeling of cells is sensitive to the temporal characteristics of the stimuli, such as the frequency of the CS. Liu et al. [4] reported that CS with frequencies ranging from 0.5 to $2 \mathrm{~Hz}$ induced SMCs to align perpendicular relative to the direction of stretch, and that 1 $\mathrm{Hz}$ induced the most rapid reorientation. In contrast, cells exposed to continuous stretch do not exhibit reorientation [4] or reorientation parallel to the direction of the CS [5]. These findings suggest that the temporal pattern of stretching is a crucial factor for CS-induced cell remodeling.

Although it remains unclear which molecule or cellular component is the primary mechanosensor for CS, one of the major candidates is focal adhesions (FAs) consisting of integrins and associated molecules. Since FAs provide the mechanical linkage between the extracellular matrix and cytoskeletons, forces would be directly exerted on FAs when cells are exposed to CS. Recent reports have shown that p130CAS [6] and talin [7] in FAs serve as primary mechanosensors, which trigger signal transduction cascades leading to alteration in cellular functions such as proliferation [8] and gene expression [9]. In addition, FAs may adapt to their mechanical environment by changing their structure and connections to cytoskeletons, and have an important role in CS-induced morphological and cytoskeletal

*Address correspondence to this author at the Graduate School of Biomedical Engineering, Tohoku University, 6-6-01 Aramaki-aoba, Aobaward, Sendai 980-8579, Japan; Tel: +81-22-795-6942; Fax: +81-22-7956943; E-mail: sato-m@ bme.tohoku.ac.jp remodeling of ECs. Previous studies using micromanipulation techniques reported accumulation of FAassociated molecules [10] and actin [11, 12] when forces were applied to FAs, and local stiffening of cells [13] was induced by continuous or cyclic force.

Numerous studies have shown responses of FAs to applied forces, yet the effects of the periodicity of the force exerted on FAs is poorly understood. This is especially true for vascular cells exposed to CS in vivo, although it is known that they respond sensitively to the temporal pattern of applied force, as mentioned above. Liu et al. [4] indicated that activation of integrin $\beta 1$, which is thought to be located upstream of signal transduction, is most effectively activated when cells are exposed to CS with a frequency of $1.25 \mathrm{~Hz}$ rather than lower or higher frequencies or continuous stretch. Matthews et al. [13] showed that locally applied periodic or continuous force induced stiffening of FAs, and suggested that this mechanical adaptation is mediated by distinct signaling pathways, depending on the periodicity of the applied forces. These findings suggest that there may be components in FAs that recognize the temporal pattern of the force. However, detailed investigations focusing on the temporal pattern of frequency or waveform have not been performed.

The present study focused on elucidating the mechanism by which FAs sense the temporal force pattern. We analyzed the reorganization of actin cytoskeletons in the vicinity of FAs exposed to various temporal patterns of cyclic forces using magnetic micro-beads and an electromagnetic tweezer. We also examined the effect of myosin activity on local force-induced actin cytoskeletal reorganization using an inhibitor and an enhancer of myosin activity, since previous studies suggested that contractile forces generated by actomyosin contribute to sensing of the temporal pattern of forces and force-induced actin reorganization [14]. 


\section{MATERIALS AND METHODS}

\section{Cell Culture}

Human umbilical vein endothelial cells (HUVECs) were isolated from umbilical cords using trypsin [15], and characterized by positively staining of an endothelial specific maker of CD31. Cells were grown in Medium 199 (Invitrogen, Carlsbad, CA, USA) supplemented with 20\% fetal bovine serum (JRH Biosciences, Lenexa, KS, USA) and $10 \mathrm{ng} / \mathrm{ml}$ basic fibroblast growth factor (Austral Biologicals, San Ramon, CA, USA). For the experiments, cells were seeded onto a glass bottom culture dish (AGC Techno Glass, Funabashi, Japan) coated with $0.1 \%$ bovine gelatin (Sigma-Aldrich, St. Louis, MO, USA). To visualize actin molecules in living cells, a plasmid encoding enhanced yellow fluorescent protein (EYFP)-actin was transfected into cells with a liposomal method using Lipofectamine LTX (Invitrogen) 2 days before the experiments. Twenty-four hours after the transfection, the medium was replaced with human endothelial serum free medium (Invitrogen) containing $1 \%$ bovine serum albumin (Wako Pure Chemical Industries, Osaka, Japan).

To inhibit the activity of myosin II in HUVECs, $10 \mu \mathrm{M}$ blebbistatin (Merck, Darmstadt, Germany), which is a selective inhibitor for non-muscle myosin II, was added to the medium $30 \mathrm{~min}$ before the experiment. Likewise, HUVECs were treated with $10 \mathrm{nM}$ calyculin A (Enzo Life Sciences, Farmingdale, NY, USA), which is a protein phosphatase inhibitor, in order to activate actomyosin contraction.

\section{Electromagnetic Tweezer System}

To apply local cyclic forces to ECs via FAs, we composed an electromagnetic tweezer (EMT) system based on a previous report [16] with some modifications. The system was built on an inverted confocal laser scanning microscope (CLSM, FV1000, Olympus, Japan) as illustrated in Fig. (1A). The EMT was attached to a hydraulic 3D micro-manipulator (MWO-3, Narishige, Tokyo, Japan) via a custom-made pipette holder (Narishige). A round permalloy

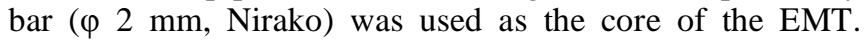
Copper wire $(\phi 0.2 \mathrm{~mm})$ was coiled around the bar with a density of 600 turns $/ \mathrm{cm}$. The tip of the bar was sharpened manually using metal files and sandpaper, and was wrapped with film sheets (\#4000, \#8000, and \#10000, Sumitomo 3M, Tokyo, Japan) to provide the micron-scale tip $(r \approx 5 \mu \mathrm{m})$.

A circuit diagram of the EMT system is shown in Fig. (1B). A multipurpose DC power supply ( $5 \mathrm{~V}, 1 \mathrm{~A})$ provided the driving current for the EMT, and the magnitude of the current was controlled with a variable resistor connected in series to the circuit. Current was switched on/off with a mechanical relay, which was controlled by a custom Labview 8.5 (National Instruments, Austin, TX, USA) program via a multi-function data acquisition unit (USB6009, National Instruments). In addition, to synchronize image acquisition with force application, the trigger signals for image acquisition by the CLSM were also controlled by the program. A nonpolarized electrolytic capacitor (NEC, 1 $\mu \mathrm{F})$ was connected in parallel with the EMT for degaussing the EMT.

To calibrate the distance-dependence of the force exerted on the beads by the EMT, the velocities of the beads driven by the magnetic force were measured in a viscous solution. The force acting on the beads was calculated based on Stokes' formula (Eq. 1):

$F=6 \pi \eta r v$,

where $\eta$ is the viscosity of the viscous solution, $r$ is the radius of the magnetic micro-bead, and $v$ is the velocity of the bead. A standard liquid for calibrating viscometers (JS500, Nippon Grease, Osaka, Japan) was used to ensure precise calibration. This liquid has a known viscosity at a given temperature $\left(\eta=149 \mathrm{mPa} \cdot \mathrm{s}\right.$ at $\left.37^{\circ} \mathrm{C}\right)$ and was utilized for the viscous solution. The distance-dependence of the force on the magnetic micro-beads $(D=4.5 \mu \mathrm{m}$, Dynabeads M450 tosyl-activated, Invitrogen) at a coil current of 300 $\mathrm{mA}$ is shown in Fig. (2). This calibration was performed each time the tip was re-polished for maintenance of the tip condition.

Residual magnetic flux of the core causes a residual pulling force during the power-off state, disturbing the cyclic force-exposure experiment. To eliminate this, we devised a degaussing method utilizing $L C R$-oscillation. When the relay was switched off, the decaying sinusoidal amplitude of voltage was applied to the coil of the EMT by the closed-
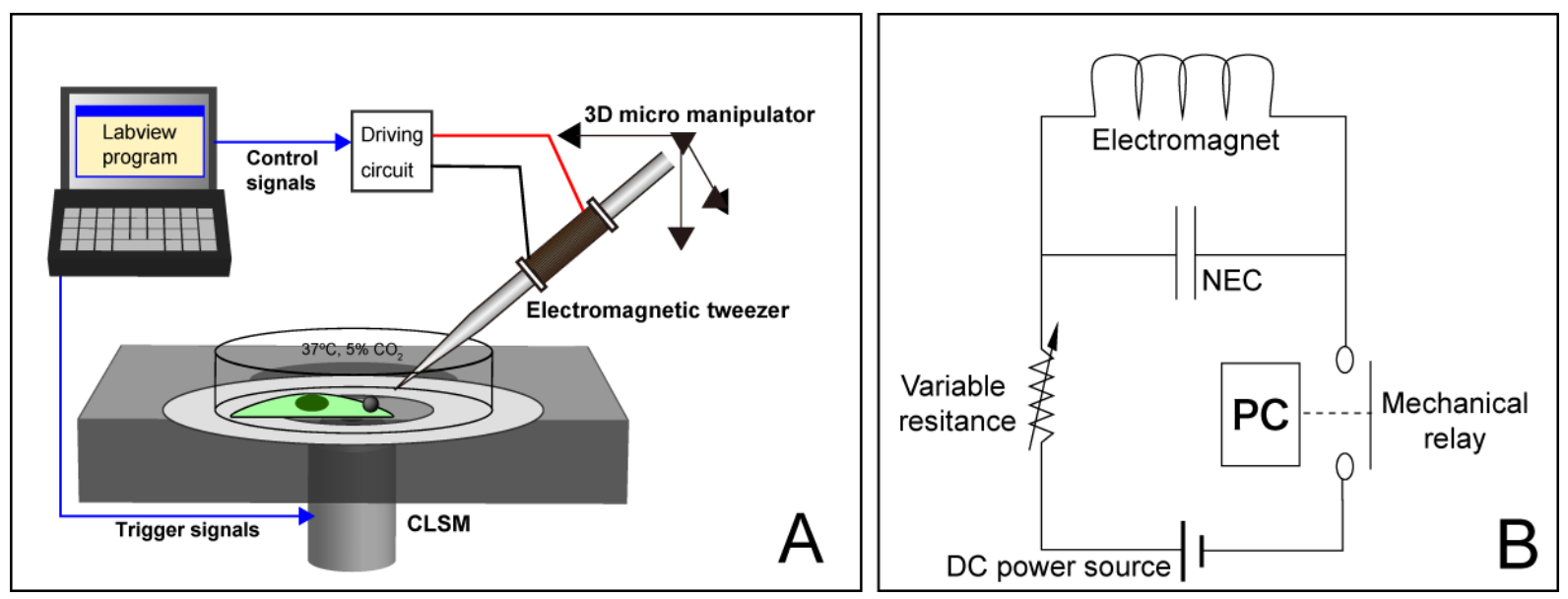

Fig. (1). Schematic overview (A) and the driving circuit (B) of the electromagnetic tweezer system for cyclic force exposure. 
loop of the $L C R$-circuit, resulting in randomization of the orientation of the permanent magnetic domain in the permalloy core. This degaussing diminished the residual force to less than $30 \mathrm{pN}$ at a distance of $30 \mu \mathrm{m}$ (Fig. 2, triangles), while a force of about $200 \mathrm{pN}$ was generated in the zero-current state in the absence of degaussing (Fig. 2, squares).

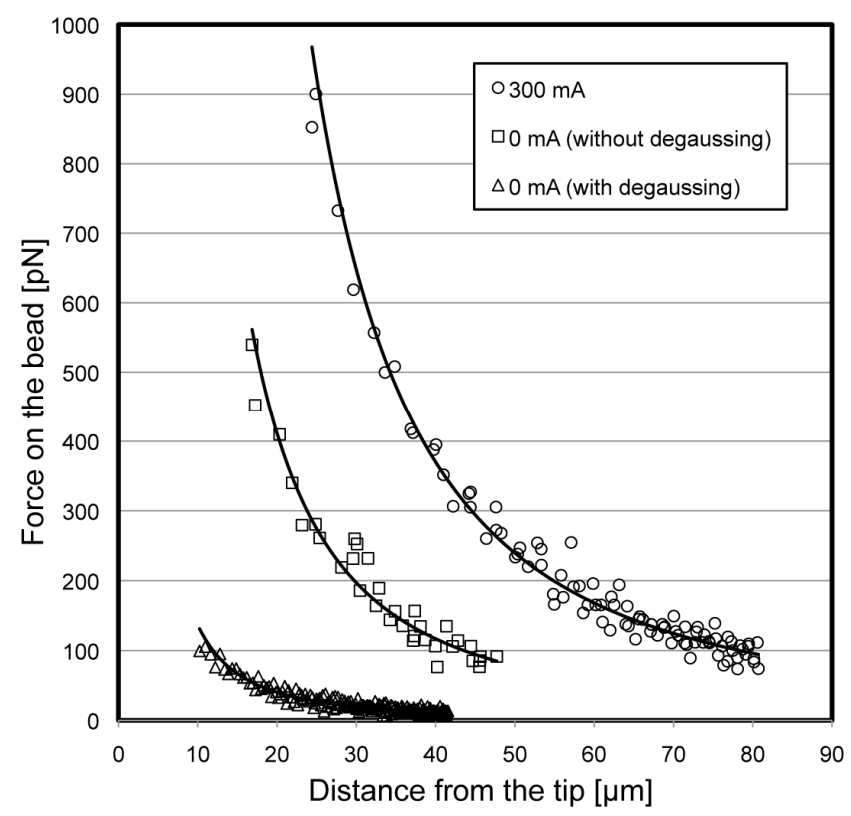

Fig. (2). The relationship between the force acting on the magnetic micro-beads and the distance from the tip of the EMT to the bead. (circle) during operation with a coil current of $300 \mathrm{~mA}$, (square) after switching off without degaussing, (triangle) after switching off with degaussing.

\section{Cyclic Force-Exposure Experiments}

Magnetic micro-beads were coated with human fibronectin (Sigma-Aldrich) according to the manufacturer's instructions. The beads were seeded on a monolayer of HUVECs on a glass bottom dish at a concentration of $1.0 \times$ $10^{4}$ beads $/ \mathrm{cm}^{2}$, resulting in about 5 attached beads/cell, followed by incubation for $15 \mathrm{~min}$ at $37^{\circ} \mathrm{C}$ to ensure integrin-mediated attachment of the beads to the cell surface. Some of the attached beads accumulated actin filaments and vinculin molecules, as shown in Fig. (3), indicating formation of FAs around the beads. Beads which had visibly accumulated EYFP-actin molecules were chosen for experiments involving the application of cyclic force via FAs. After incubation, unattached beads were removed by rinsing the cells twice with medium.

A cyclic force was applied to individual beads on the EC using the EMT system. The distance between the tip and the bead was maintained at $40-50 \mu \mathrm{m}$ in order to apply a pulling force of $300 \mathrm{pN}$ on the bead, according to the force calibration curve (Fig. 2). The change in the distance between the bead and the tip affects the magnitude of value. However, in the present study, the displacement of the beads during force application was less than $1 \mu \mathrm{m}$, resulting in the change in the force of about $10 \mathrm{pN}$, which is smaller than forces required for the unbinding or unfolding of a single molecule [17] and would not have a major effect on force-

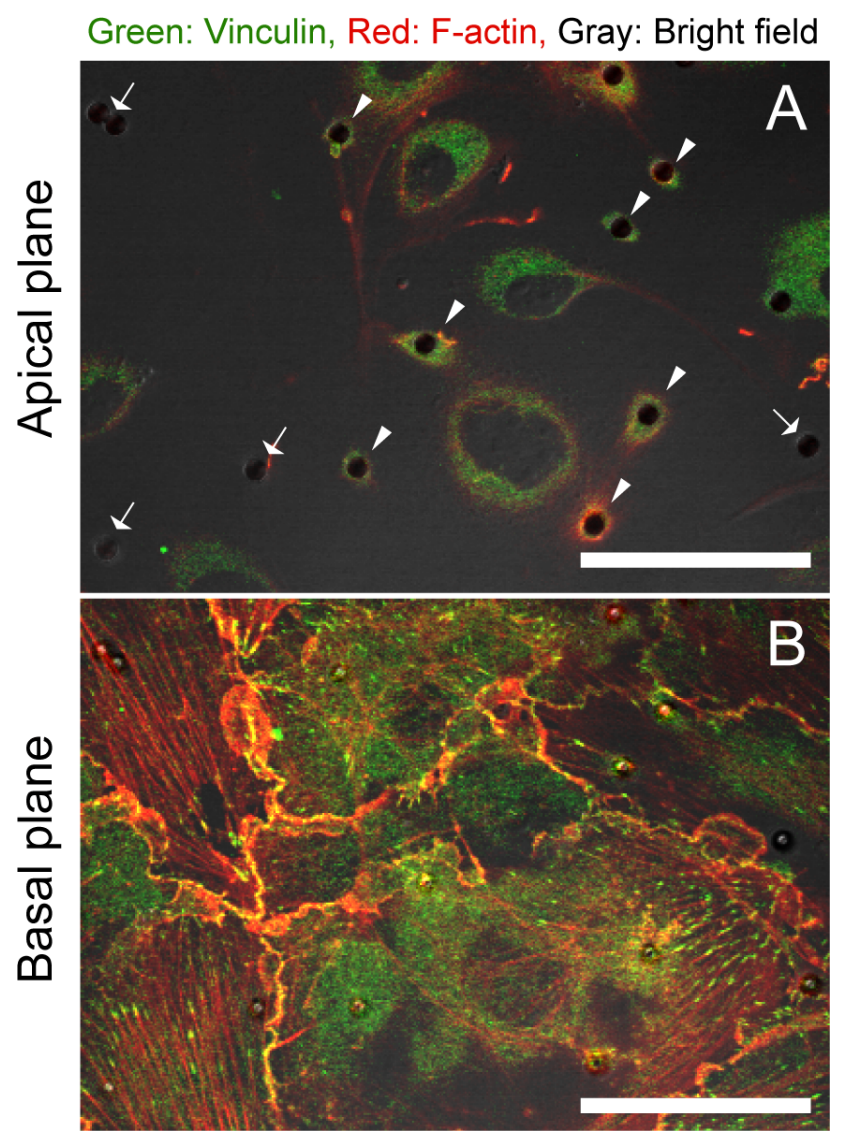

Fig. (3). Merged images of a confocal fluorescence image and a bright field image. (green) vinculin, (red) F-actin. (A) an apical plane focused on the magnetic micro-beads on the cell surface, (B) a basal plane focused on the basal FAs. (arrowheads) beads with accumulated vinculin molecules and actin filaments, (arrows) beads without accumulated molecules.

induced actin remodeling. The experimental regime for cyclic force loading is shown in Fig. (4). A square wave current $(300 \mathrm{~mA})$ at a frequency of $0.1,0.5,1,2$ or $10 \mathrm{~Hz}$ with a duty cycle of 0 (static), $5,25,50,75,95$ or $100 \%$ (continuous loading) was applied to the coil. Fluorescence images of EYFP-actin were obtained with a CLSM and 60x objective (UPLSAPO, NA $=1.35$, Olympus) every $2.5 \mathrm{~s}$ until $2 \mathrm{~min}$ after initiation of the force exposure. For cells pretreated with blebbistatin or calyculin A, the experiments were performed in medium containing the same concentration of the reagents. During the experiments, the temperature and gas conditions were maintained at $37^{\circ} \mathrm{C}$ and $5 \% \mathrm{CO}_{2}$, respectively, using a $\mathrm{CO}_{2}$ incubator mounted on the stage of the CLSM.

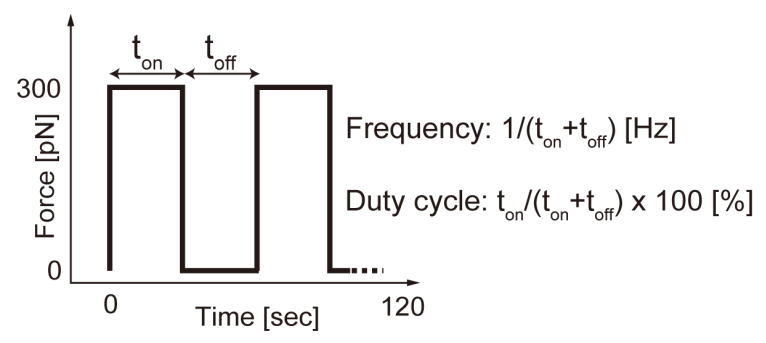

Fig. (4). Schematic diagram of the experimental regime for cyclic force exposure. 

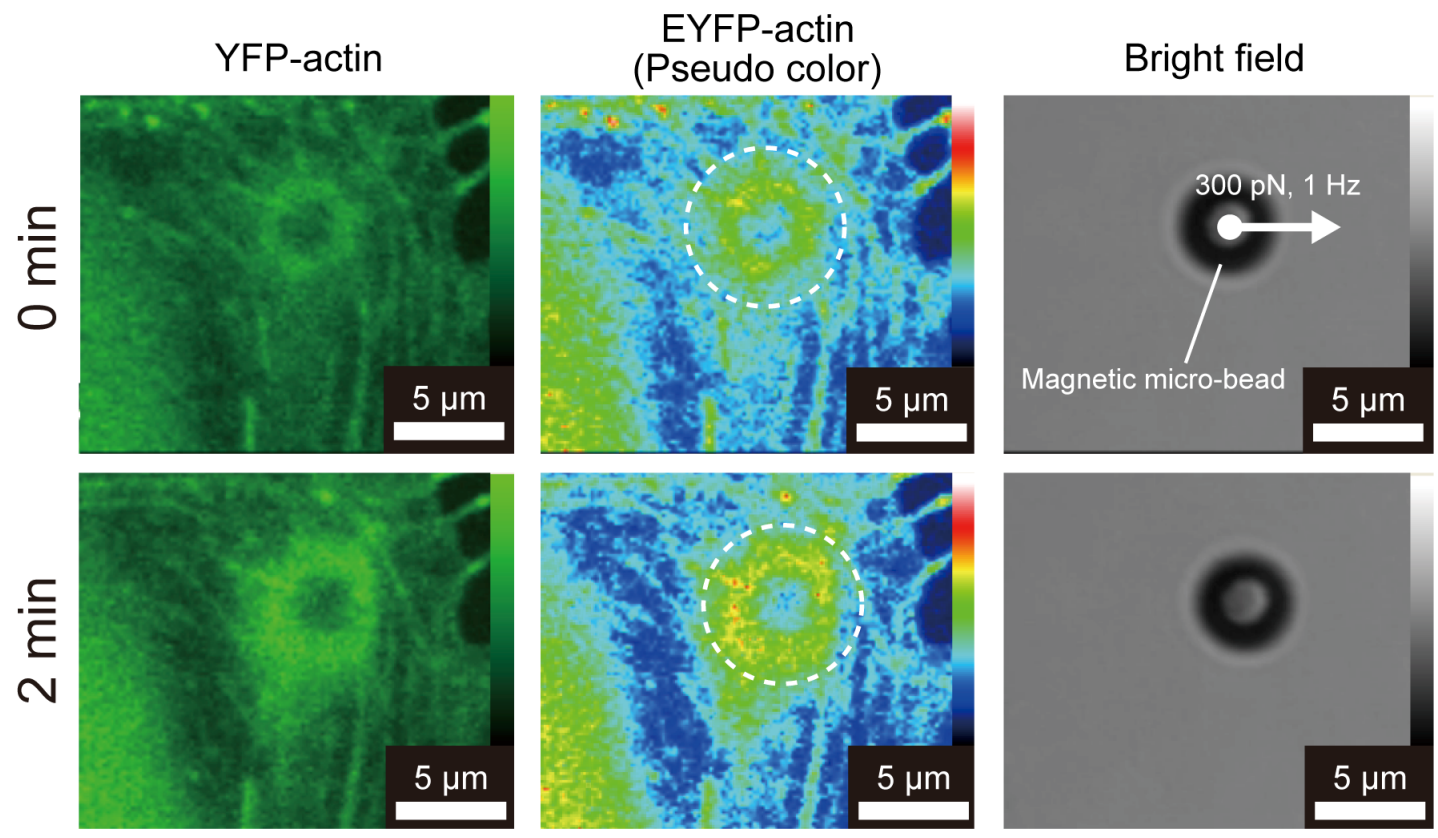

Fig. (5). Representative images of actin recruitment around a bead before and after loading of a cyclic force with a frequency of $1 \mathrm{~Hz}$, duty cycle of $50 \%$, and amplitude of $300 \mathrm{pN}$. (dotted circles) the regions subjected to image analysis. Warmer color in pseudo color images indicates the higher fluorescence intensity of YFP-actin.

\section{Image Analysis and Statistical Analysis}

To quantitatively evaluate the recruitment of actin molecules around the beads, we measured the coefficient of variation of pixel intensities in a $7-\mu \mathrm{m}$ diameter circular region centered on the bead (Fig. 5, dotted circles), in accordance with a previous report [18]. When actin molecules are recruited around a bead, pixels in the region exhibit higher variability in fluorescence intensities, whereas lower variability is obtained when actin molecules are more evenly distributed in the region. The standard deviation of fluorescence intensities in the region was therefore defined as the recruitment index (RI) for evaluations of actin recruitment around a bead. This parameter can express actin recruitment with avoiding the effect of change in fluorescent intensity due to photobleaching. RI was calculated with the image analysis software, ImageJ 1.40 (National Institutes of Health, Bethesda, MD, USA), and Excel for Mac 2004 (Microsoft, Redmond, WA, USA). Values of RI obtained before and after cyclic force-exposure were compared using the Wilcoxon signed rank test using a language and environment for statistical computing, $\mathrm{R}$ ( $\mathrm{R}$ Development Core Team, Vienna, Austria). $p$ values less than 0.05 were considered to be significant.

\section{RESULTS}

\section{Effect of Frequency of Cyclic Force on Actin Recruitment}

Representative fluorescence images of EYFP-actin before and after $2 \mathrm{~min}$ exposure to a $1-\mathrm{Hz}$ cyclic force are shown in Fig. (5). An increase in fluorescence intensity of EYFP-actin recruited around the bead was observed after exposure to the cyclic force. Relative changes in RI $2 \mathrm{~min}$ after exposure to the cyclic force at various frequencies (the duty cycle was set at 50\%.) are shown in Fig. (6A). A significant increase in RI was observed at 1 and $2 \mathrm{~Hz}$, which are in the physiological range of frequencies for ECs, whereas a significant change in RI was not observed at lower frequencies $(0.1$ and $0.5 \mathrm{~Hz})$ or higher frequency $(10 \mathrm{~Hz})$.

\section{Effect of Duty Cycle of Cyclic Force on Actin Recruitment}

The relative changes in RI induced by cyclic force exposure with various duty cycles at a frequency of $1 \mathrm{~Hz}$ are shown in Fig. (6B). While no significant changes in actin recruitment were observed when the force was applied with lower duty cycles $(0-5 \%)$ or higher duty cycles, including continuous loading (75-100\%), cyclic forces with duty cycles of 25 and $50 \%$ induced significant increases in actin recruitment around the beads.

\section{Effect of Myosin Activity on Frequency Dependence in Actin Recruitment}

Actin recruitment due to cyclic force application to FAs in ECs treated with blebbistatin or calyculin A is shown in Fig. (7). Cyclic forces did not induce a significant increase in RI in ECs treated with blebbistatin, while continuous forceexposure significantly increased RI. On the other hand, the increasing tendency in RI was observed for ECs treated with calyculin A after exposure to both continuous and cyclic stretches and statistically significant differences were detected in continuous and $1-\mathrm{Hz}$ forces.

\section{DISCUSSION}

A previous study reported that a relatively long time $(\sim 15$ min) is required to achieve actin recruitment around FAs when continuous forces are applied [11]. In contrast, cyclic forces at 1 and $2 \mathrm{~Hz}$ induced actin recruitment within $2 \mathrm{~min}$ in the present study. This result suggests that the periodicity of loading and relaxing the applied force may enhance the mechanically-induced cytoskeletal reorganization mediated by FAs. In addition, the result is consistent with a previous 

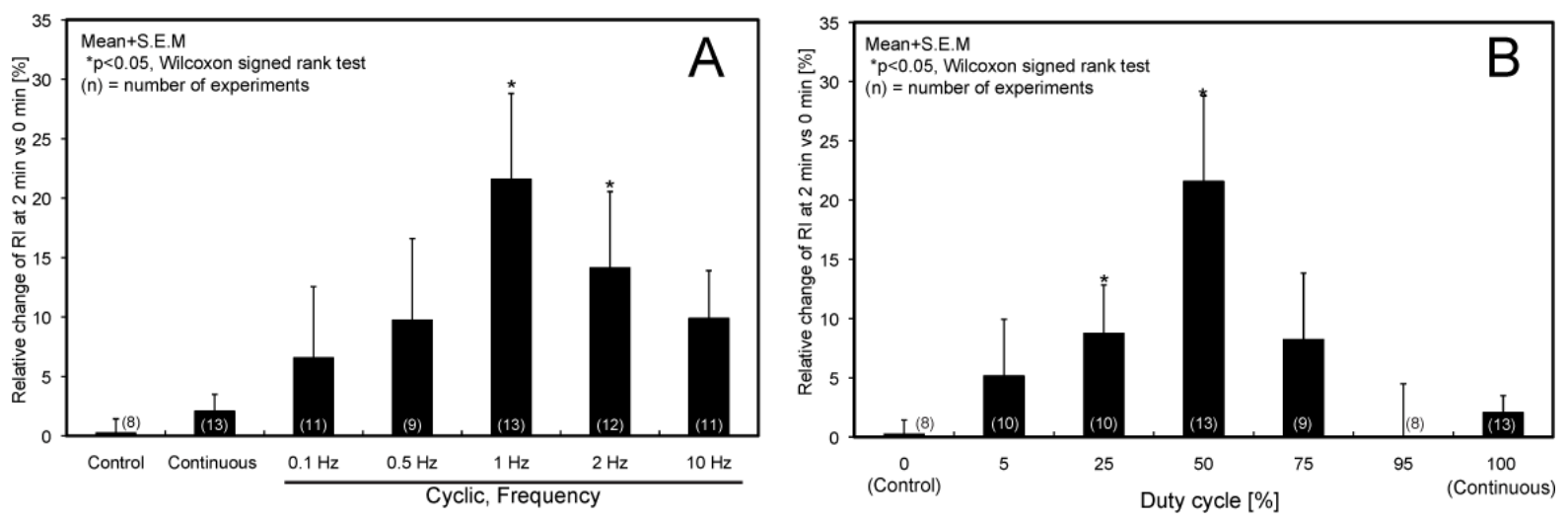

Fig. (6). Relative increase of RI 2 min after initiation of the local force to FAs with various frequencies (A) and duty cycles (B). Note that duty cycles of 0 and $100 \%$ in $(\mathbf{B})$ correspond to "Control" and "Continuous" in (A), respectively.

result showing that CS at a frequency of $1.25 \mathrm{~Hz}$ is the most effective frequency for cell alignment and integrinactivation, compared to other frequencies or constant stretching [4]. However, it remains unclear why a higher frequency of $10 \mathrm{~Hz}$ is less effective for actin recruitment than 1 or $2 \mathrm{~Hz}$. We speculated that the duration of forceloading in each cycle of stimulation at higher frequency may be insufficient to induce integrin activation because FAs are known to exhibit creep behavior in response to an applied step force $[19,20]$. The time constant for creep deformation of FAs, which is the time required for the transition from elastic to viscous behavior, was reported to be in the range 0.1-0.5 s $[19,20]$. Interestingly, this time constant corresponds to the duration of force loading or relaxation in a single cycle of $1-$ or $2-\mathrm{Hz}$ exposure in the present study. Hence, it is speculated that repetition of a sufficient loading duration, which may lead to deformation of FAs in each cycle, is crucial for cyclic force-induced actin remodeling. This idea is supported by our finding that a $1-\mathrm{Hz}$ cyclic force with a duty cycle of $5 \%$, which has the same loading period of $0.05 \mathrm{~s}$ as a $10-\mathrm{Hz}$ force, did not induce a significant increase in actin recruitment.

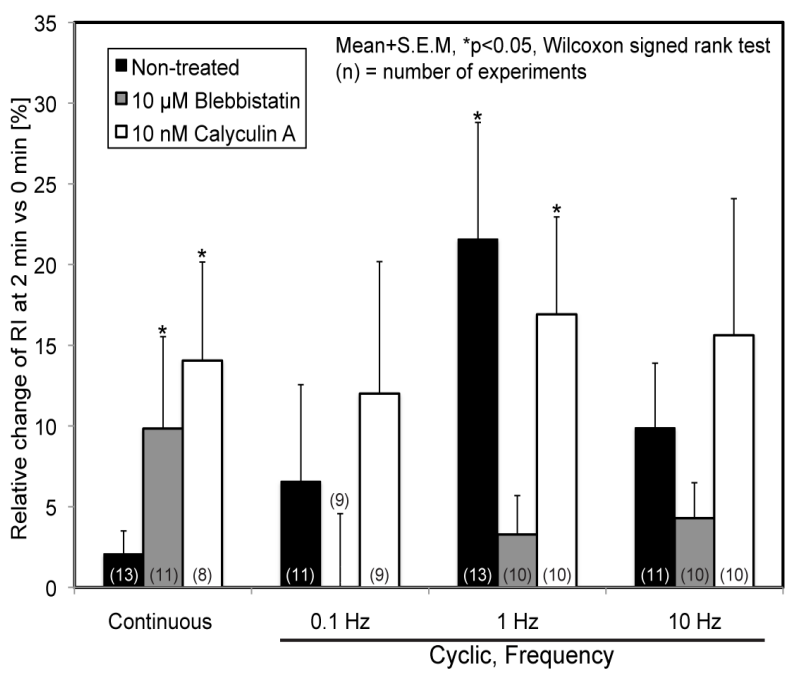

Fig. (7). Relative increase of RI 2 min after initiation of the local force to FAs with various frequencies in ECs treated with blebbistatin or calyculin A.
A recent report showed that the duration of force-loading affects the unfolding of talin molecules, leading to binding of vinculin molecules [7]. The dependency of cytoskeletal remodeling on the temporal pattern of mechanical loading may, therefore, be due to dynamic conformational changes in mechanosensitive molecules. In addition, both the loading and relaxation phases may play an important role in actin remodeling because the $1-\mathrm{Hz}$ force with higher duty cycles (75 and 95\%) did not induce a significant increase in actin recruitment in the present study. While many studies have focused on the effect of the force-loading phase on mechanosensitive molecules, the result of this study indicates the importance of focusing on the effect of relaxation from force in order to better understand mechanotransduction.

The present study showed that inhibition of actomyosin activity with blebbistatin suppressed cyclic force-induced actin recruitment. Since the development of FAs in the static condition is known to be regulated by the actomyosin contractile force of actin cytoskeletons connected to the FAs [10], it is possible that insufficient development of FAs around the beads is caused by a decrease in myosin activity. In the present study, we applied local cyclic or continuous forces to beads that accumulated actin molecules prior to force exposure. We confirmed that RI decreased $(p<0.05)$ or increased $(p<0.01)$ following treatment with blebbistatin or calyculin A, respectively, compared to untreated cells under static conditions (data not shown), indicating that the development of FAs around the beads was affected by the modulation of myosin activity. In ECs treated with calyculin $\mathrm{A}$, an increase in actin recruitment was observed when both continuous and cyclic forces were applied, and dependence on the temporal pattern of the applied forces was diminished. Calyculin A is one of protein phosphatase inhibitors and known to elevate phosphorylation of myosin light chain, resulting in the increase in contraction of actomyosin. Since it is reported that development of FAs is regulated by actomyosin contractile force [10], and calyculin A treatment caused the increase in accumulation of actin molecules around the beads under static conditions, FAs would develop excessively around the beads before the force application, which may lead to increased actin recruitment regardless of the periodicity of the applied forces. 


\section{CONCLUSION}

In the present study, the effect of the temporal pattern of forces applied to FAs on actin reorganization was investigated. It was found that actin recruitment was effectively induced by cyclic forces with frequencies of 1-2 $\mathrm{Hz}$ and with duty cycles of $25-50 \%$. These results suggest the importance of the periodicity of forces at both the loading and relaxation phases on mechanically-induced cytoskeletal remodeling. In addition, the observed modification of myosin activity suggested that sensing of the temporal pattern by FAs depends on the development of FAs mediated by actomyosin contractile activity.

\section{ACKNOWLEDGEMENTS}

The Authors thank Dr. Ikuo Takahashi for kindly providing human umbilical cords with the informed consent of the donors. This work was in part supported financially by Grants-in-Aid for Scientific Research from the Ministry of Education, Culture, Sports, Science and Technology (MEXT) in Japan (Nos. 20001007 and 21-3835).

\section{REFERENCES}

[1] V. P. Shirinsky, A. S. Antonov, K. G. Birukov, A. V. Sobolevsky, Y. A. Romanov, N. V. Kabaeva, G. N. Antonova, and V. N. Smirnov, "Mechano-chemical control of human endothelium orientation and size," J. Cell Biol., vol. 109, pp. 331-9, Jul 11989.

[2] T. Takemasa, K. Sugimoto, and K. Yamashita, "Amplitudedependent stress fiber reorientation in early response to cyclic strain," Exp. Cell Res., vol. 230, pp. 407-10, Feb 11997.

[3] K. Naruse, T. Yamada, and M. Sokabe, "Involvement of SA channels in orienting response of cultured endothelial cells to cyclic stretch," Am. J. Physiol., vol. 274, pp. H1532-8, May 11998.

[4] B. Liu, M. J. Qu, K. R. Qin, H. Li, Z. K. Li, B. R. Shen, and Z. L. Jiang, "Role of cyclic strain frequency in regulating the alignment of vascular smooth muscle cells in vitro," Biophys. J., vol. 94, pp. 1497-507, Feb 152008.

[5] A. Sasamoto, M. Nagino, S. Kobayashi, K. Naruse, Y. Nimura, and M. Sokabe, "Mechanotransduction by integrin is essential for IL-6 secretion from endothelial cells in response to uniaxial continuous stretch," Am. J. Physiol. Cell Physiol., vol. 288, pp. C1012-22, May 12005.

[6] Y. Sawada, M. Tamada, B. J. Dubin-Thaler, O. Cherniavskaya, R. Sakai, S. Tanaka, and M. P. Sheetz, "Force sensing by mechanical extension of the Src family kinase substrate p130Cas," Cell, vol. 127, pp. 1015-26, Dec 12006.

[7] A. del Rio, R. Perez-Jimenez, R. Liu, P. Roca-Cusachs, J. M. Fernandez, and M. P. Sheetz, "Stretching single talin rod molecules activates vinculin binding," Science, vol. 323, pp. 638-41, Jan 30 2009.

[8] C. D. Shrader, H. G. Ressetar, J. Luo, E. V. Cilento, and F. D. Reilly, "Acute stretch promotes endothelial cell proliferation in wounded healing mouse skin," Arch. Dermatol. Res., vol. 300, pp. 495-504, Oct 2008.

[9] S. R. Frye, A. Yee, S. G. Eskin, R. Guerra, X. Y. Cong, and L. V. McIntire, "CDNA microarray analysis of endothelial cells subjected to cyclic mechanical strain: importance of motion control," Physiol. Genomics., vol. 21, pp. 124-130, Mar 212005.

[10] C. G. Galbraith, K. M. Yamada, and M. P. Sheetz, "The relationship between force and focal complex development," $J$. Cell Biol., vol. 159, pp. 695-705, Nov 252002.

[11] D. Icard-Arcizet, O. Cardoso, A. Richert, and S. Hénon, "Cell stiffening in response to external stress is correlated to actin recruitment," Biophys. J., vol. 94, pp. 2906-13, Apr 12008.

[12] L. Deng, N. J. Fairbank, B. Fabry, P. G. Smith, and G. N. Maksym, "Localized mechanical stress induces time-dependent actin cytoskeletal remodeling and stiffening in cultured airway smooth muscle cells," Am. J. Physiol. Cell Physiol., vol. 287, pp. C440-8, Aug 12004.

[13] B. D. Matthews, D. R. Overby, R. Mannix, and D. E. Ingber, "Cellular adaptation to mechanical stress: role of integrins, Rho, cytoskeletal tension and mechanosensitive ion channels," J. Cell Sci., vol. 119, pp. 508-18, Feb 12006.

[14] J. H. Wang, P. Goldschmidt-Clermont, and F. C. Yin, "Contractility affects stress fiber remodeling and reorientation of endothelial cells subjected to cyclic mechanical stretching," Ann. Biomed. Eng., vol. 28, pp. 1165-71, 2000.

[15] N. Sakamoto, T. Ohashi, and M. Sato, "Effect of magnetic field on nitric oxide synthesis of cultured endothelial cells," Int. J. Appl. Electromagn. Mech., vol. 14, pp. 317-322, 2001.

[16] B. D. Matthews, D. A. LaVan, D. R. Overby, J. Karavitis, and D. E. Ingber, "Electromagnetic needles with submicron pole tip radii for nanomanipulation of biomolecules and living cells," Appl. Phys. Lett., vol. 85, pp. 2968-2970, Oct 42004.

[17] Z. Sun, L. A. Martinez-Lemus, A. Trache, J. P. Trzeciakowski, G. E. Davis, U. Pohl, and G. A. Meininger, "Mechanical properties of the interaction between fibronectin and alpha5beta1-integrin on vascular smooth muscle cells studied using atomic force microscopy," Am. J. Physiol. Heart Circ. Physiol., vol. 289, pp. H2526-35, Dec 12005.

[18] R. S. Ozer, and S. Halpain, "Phosphorylation-dependent localization of microtubule-associated protein MAP2c to the actin cytoskeleton," Mol. Biol. Cell, vol. 11, pp. 3573-87, Oct 12000.

[19] A. R. Bausch, F. Ziemann, A. A. Boulbitch, K. Jacobson, and E. Sackmann, "Local measurements of viscoelastic parameters of adherent cell surfaces by magnetic bead microrheometry," Biophys. J., vol. 75, pp. 2038-49, Oct 11998.

[20] B. D. Matthews, D. R. Overby, F. J. Alenghat, J. Karavitis, Y. Numaguchi, P. G. Allen, and D. E. Ingber, "Mechanical properties of individual focal adhesions probed with a magnetic microneedle," Biochem. Biophys. Res. Commun., vol. 313, pp. 758-64, Jan 16 2004.

() Ueki et al.; Licensee Bentham Open.

This is an open access article licensed under the terms of the Creative Commons Attribution Non-Commercial License (http://creativecommons.org/licenses/by-nc/3.0/) which permits unrestricted, non-commercial use, distribution and reproduction in any medium, provided the work is properly cited. 